\title{
FROM NEWTON TO NAVIER-STOKES, OR HOW TO CONNECT FLUID MECHANICS EQUATIONS FROM MICROSCOPIC TO MACROSCOPIC SCALES
}

\author{
ISABELLE GALLAGHER
}

\begin{abstract}
In this survey we present an overview of some mathematical results concerning the passage from the microscopic description of fluids via Newton's laws to the macroscopic description via the Navier-Stokes equations.
\end{abstract}

\section{INTRODUCTION AND PLAN OF THE SURVEY}

The mathematical description of fluids is an intricate process which depends on the scale of observation:

- at a microscopic level one sees atoms, and the gas may be described by a classical mechanics picture via Newton's ordinary differential equations (ODEs), which is presented in Section 2.1; and

- at a macroscopic scale one is interested in an average behavior modeled by partial differential equations (PDEs) such as the Navier-Stokes equations, which are described in Section 2.2

- an intermediate regime, called mesoscopic scale, can also be used, and the corresponding Boltzmann PDE is presented in Section 2.3.

Our goal in this survey is to explain how these apparently very different descriptions (ODEs vs PDEs, reversible vs irreversible dynamics...) can be related one to the other from a mathematical point of view: this question goes back to Hilbert (see Section 2.4) and has known quite a lot of progress in recent years; we refer for instance, among many others, to [41,42,70 72. In Section 3 we present some mathematical attempts to justify the passage from one scale to the other by a limiting process, and we show the limitations of those approaches which prevents solving the full problem: justifying nonlinear fluid mechanics PDEs from the microscopic ODEs. Section 4 describes one situation where the full problem does have an answer, in a linear setting. Finally, in Section 5 a few questions are presented.

\section{Microscopic, Mesoscopic, AND MACROsCopiC SCALES IN FLUIDS}

\subsection{Newton: A microscopic point of view.}

2.1.1. The equations. A gas is made of a very large number of particles evolving and interacting in a $d$-dimensional space domain. Throughout this survey we assume that the space dimension is $d \geq 2$, and we denote by $N \gg 1$ the number of particles. Typically $N$ is larger than the Avogadro number $6.02 \cdot 10^{23}$.

Received by the editors June 11, 2018.

2010 Mathematics Subject Classification. Primary 76D05, 82B40, 82C22.

Key words and phrases. Kinetic equations, fluid dynamics, particle systems, Boltzmann equation, Navier-Stokes equation, Boltzmann-Grad limit, low density limit. 
A number of simplifying assumptions will be made throughout this survey, concerning the space domain, the types of particles considered, and their interactions. These are listed below, and comments on these assumptions can be found in the concluding section:

- the particles are all identical spheres of mass 1 and diameter $\varepsilon>0$;

- the particles evolve in a periodic box of size 1 denoted $\mathbb{T}^{d}:=[0,1]^{d}$;

- the particles interact elastically at each binary collision, and there is no other type of interaction nor forcing.

We label by integers $i \in\{1, \ldots, N\}$ each particle - note that the particles are indistinguishable, so this labeling is arbitrary and all the functions we shall consider will be symmetric with respect to permutations of the labels. We denote by $\left(x_{i}, v_{i}\right) \in \mathbb{T}^{d} \times \mathbb{R}^{d}$ the position and velocity of particle $i$ for $1 \leq i \leq N$. Due to the fact that the particles are hard-spheres, the nonoverlapping condition holds

$$
\left|x_{i}-x_{j}\right|>\varepsilon \text {. }
$$

We denote by $Z_{N}:=\left(z_{1}, \ldots, z_{N}\right)$ the set of configurations of the particles, with $z_{i}:=$ $\left(x_{i}, v_{i}\right)$ for each particle. We also denote in the following by $X_{N}:=\left(x_{1}, \ldots, x_{N}\right)$ the set of positions and by $V_{N}:=\left(v_{1}, \ldots, v_{N}\right)$ the set of velocities of the particles. The positions and velocities of the system of $N$ particles obey the Newton laws, which are the following equations of motion

$$
\forall i \in[1, \ldots, N], \quad \frac{d x_{i}(t)}{d t}=v_{i}(t), \quad \frac{d v_{i}(t)}{d t}=0,
$$

provided that the exclusion condition $\left|x_{i}(t)-x_{j}(t)\right|>\varepsilon$ is satisfied for all $j \neq i$. Therefore, the flow takes place in the domain

$$
\mathcal{D}_{N}^{\varepsilon}:=\left\{Z_{N} \in \mathbb{T}^{d N} \times \mathbb{R}^{d N} \quad \forall i \neq j,\left|x_{i}-x_{j}\right|>\varepsilon\right\} .
$$

We further have to prescribe a reflection condition at the boundary of $\mathcal{D}_{N}^{\varepsilon}$ : if there exist $j \neq i$ such that $\left|x_{i}-x_{j}\right|=\varepsilon$, then the incoming velocities $v_{i}^{i n}, v_{j}^{i n}$ are related to the outgoing velocities $v_{i}^{\text {out }}, v_{j}^{\text {out }}$ through the relations

$$
\begin{aligned}
& v_{i}^{\text {in }}=v_{i}^{\text {out }}-\nu^{i, j} \cdot\left(v_{i}^{\text {out }}-v_{j}^{\text {out }}\right) \nu^{i, j}, \\
& v_{j}^{\text {in }}=v_{j}^{\text {out }}+\nu^{i, j} \cdot\left(v_{i}^{\text {out }}-v_{j}^{\text {out }}\right) \nu^{i, j},
\end{aligned}
$$

where

$$
\nu^{i, j}:=\frac{x_{i}-x_{j}}{\left|x_{i}-x_{j}\right|}
$$

Note that incoming velocities are defined by the fact that

$$
\nu^{i, j} \cdot\left(v_{i}^{i n}-v_{j}^{i n}\right)<0,
$$

meaning that incoming velocities are precollisional, and similarly

$$
\nu^{i, j} \cdot\left(v_{i}^{\text {out }}-v_{j}^{\text {out }}\right)>0,
$$

meaning that outgoing velocities are postcollisional. Note that this is a Hamiltonian system and the Lebesgue measure in the phase space is preserved by the flow. Moreover, the momentum and energy are conserved through a collision (see Figure 1),

$$
\begin{aligned}
v_{i}^{\text {in }}+v_{i}^{\text {in }} & =v_{i}^{\text {out }}+v_{j}^{\text {out }}, \\
\left|v_{i}^{\text {in }}\right|^{2}+\left|v_{i}^{\text {in }}\right|^{2} & =\left|v_{i}^{\text {out }}\right|^{2}+\left|v_{j}^{\text {out }}\right|^{2} .
\end{aligned}
$$




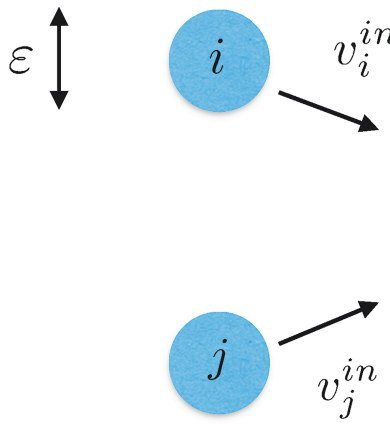

Before

collision

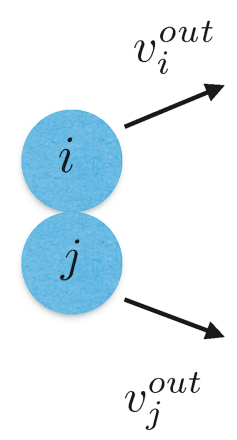

At collision
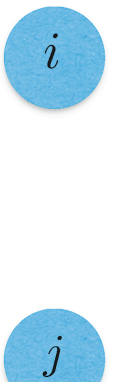

After collision

Figure 1. Collision of two particles

2.1.2. Solving the Newton equations. It is not obvious to check that the Newton equations (2.1) -(2.3) define global dynamics. Indeed this is not a simple consequence of the Cauchy-Lipschitz theorem since the boundary condition is not smooth, and it is even not defined for all configurations. We call a trajectory pathological when either

- there exists a collision involving more than two particles, or the collision is grazing (meaning that $\nu^{i, j} \cdot\left(v_{i}^{i n}-v_{j}^{i n}\right)=0$ ), hence the boundary condition is not well-defined; or

- there are an infinite number of collisions in finite time so the dynamics cannot be globally defined.

In [1, 2, it is proved that outside a negligible set of initial data there are no pathological trajectories.

Proposition 2.1. Let $N, \varepsilon$ be fixed. The set of initial configurations leading to a pathological trajectory is of measure zero in $\mathbb{T}^{d N} \times \mathbb{R}^{d N}$.

Sketch of proof. Let us recall briefly the proof given in [36, following [1,2]. For any integer $s \in \mathbb{N}^{*}$ and any $R>0$, we denote

$$
B_{R}^{s}:=\left\{V_{s} \in \mathbb{R}^{d s},\left|V_{s}\right| \leq R\right\},
$$

where $|\cdot|$ is the euclidean norm. Now let us fix $R>0, \delta<\varepsilon / 2$ (recall that $\varepsilon$ is the diameter of the particles), and $t>0$, and let us assume $t / \delta$ is an integer. Then it is easy to see that the set

$$
\left\{Z_{N} \in B_{R}^{N} \times B_{R}^{N} / \text { one particle will collide with two others on the time }[0, \delta]\right\}
$$

has measure smaller than $C(N, \varepsilon, R) \delta^{2}$. Moreover, up to removing a measure zero set of initial data, each collision on $[0, \delta]$ is nongrazing. We can repeat this argument starting again at time $\delta$ since the measure is invariant by the flow, so repeating the procedure $t / \delta$ times produces a subset $I_{\delta}(t, R)$ of $B_{R}^{N} \times B_{R}^{N}$, of measure

$$
\left|I_{\delta}(t, R)\right| \leq C(N, R, t, \varepsilon) \delta
$$


such that for any initial configuration in $B_{R}^{N} \times B_{R}^{N}$ outside that set, the flow is welldefined up to time $t$. The intersection $I(t, R):=\bigcap_{\delta>0} I_{\delta}(t, R)$ is of measure zero, and any initial configuration in $B_{R}^{N} \times B_{R}^{N}$ outside $I(t, R)$ generates a well-defined flow until time $t$. Finally, any initial configuration in $\mathbb{T}^{d N} \times \mathbb{R}^{d N}$ outside $I:=$ $\bigcup_{n} I\left(t_{n}, R_{n}\right)$, where $t_{n}$ and $R_{n}$ go to infinity, generates a globally defined flow. The proposition is proved.

\subsection{Euler and Navier-Stokes: A macroscopic point of view.}

2.2.1. The equations. The history of the mathematical study of fluids goes back many centuries, but one can probably date to the mid-eighteenth century the first equations describing fluid flows. In 1748, the Academy of Sciences in Berlin announced a Mathematics Prize for 1750, regarding the resistance of fluids when a rigid body is immersed in the fluid. J. d'Alembert took part in the competition and submitted a manuscript [3] which was revolutionary in many aspects: in particular for the first time the movement of a fluid was described by partial differential equations acting on the velocity field of the fluid. This model was close to being correct, and the final equations were coined by L. Euler in 34 - if $u$ is the time- and space-dependent, three-component vector field denoting the velocity of the fluid, then it solves the following system of PDEs:

$$
\left\{\begin{array}{l}
\partial_{t} u+u \cdot \nabla u=-\nabla p \\
\operatorname{div} u=0
\end{array}\right.
$$

where $p$ is the pressure of the fluid, also an unknown, guaranteeing that the fluid remains incompressible (meaning div $u=0$ ) for all times. The first equation in (E) translates the conservation of momentum, while the second one stands for the conservation of mass.

However, d'Alembert quickly realized that a solid body placed in a fluid whose velocity satisfies those equations can evolve without suffering any resistance, which is obviously contrary to intuition and physical experiments. This is known as d'Alembert's paradox. To understand why a solid body is submitted in general to a force tending to slow it down, one needs to take into account friction phenomena, at a molecular level: as it evolves, the fluid will have a tendency to dissipate energy under the form of heat. This phenomenon is absent from the Euler equations. In 1820, L. Navier 61 had the idea of introducing an additional term to the Euler equations, intended to represent this dissipation of energy. Followed, among others, by G. Stokes in 1845 (75]), Navier suggested the following model to describe the evolution of a viscous fluid:

$$
\left\{\begin{array}{l}
\partial_{t} u+u \cdot \nabla u-\nu \Delta u=-\nabla p \\
\operatorname{div} u=0
\end{array}\right.
$$

The parameter $\nu>0$ is the fluid's viscosity, and it measures the discrepancy between a viscous and a perfect fluid.

2.2.2. Solving the Navier-Stokes equations. The question of the resolution of (NS) is not the topic of this survey. It is however useful to go rapidly through its main properties and recall the main results concerning its solutions. The most important property of (NS) relates to the conservation of energy. Formally, if one computes the scalar product of $u$ with the momentum conservation equation in (NS), noticing 
that

$$
(u \cdot \nabla u \mid u)_{L^{2}}=-\frac{1}{2} \int \operatorname{div} u|u|^{2} d x=0 \quad \text { and } \quad(\nabla p \mid u)_{L^{2}}=-(p \mid \operatorname{div} u)_{L^{2}}=0,
$$

one finds that

$$
\frac{1}{2} \frac{d}{d t}\|u(t)\|_{L^{2}}^{2}+\nu\|\nabla u(t)\|_{L^{2}}^{2}=0 .
$$

After integration in time this implies that for all $t \geq 0$, the solution $u(t)$ associated with the initial data $u_{0}$ satisfies (formally)

$$
\frac{1}{2}\|u(t)\|_{L^{2}}^{2}+\nu \int_{0}^{t}\left\|\nabla u\left(t^{\prime}\right)\right\|_{L^{2}}^{2} d t^{\prime}=\frac{1}{2}\left\|u_{0}\right\|_{L^{2}}^{2} .
$$

This implies in particular that the map $t \mapsto\|u(t)\|_{L^{2}}$ is decreasing. Moreover. this equality shows the smoothing effect induced by viscosity since as soon as the initial data is of finite energy (meaning that it lies in $L^{2}$ ), the solution is instantaneously smoother in the sense that $\nabla u$ belongs to $L^{2}\left(\mathbb{R}^{+} ; L^{2}\right)$.

Another important property of (NS) is its scale invariance: if $u$ solves (NS) on $[0, T] \times \mathbb{R}^{d}$, then for any $\lambda>0$

$$
u_{\lambda}(t, x):=\lambda u\left(\lambda^{2} t, \lambda x\right)
$$

solves $(\mathrm{NS})$ on $\left[0, \lambda^{-2} T\right] \times \mathbb{R}^{d}\left(\right.$ for the rescaled data $\lambda u_{0}(\lambda x)$ ).

The issue is now to use those properties to solve the equations: Given an initial data $u_{0}$, is there a solution to (NS) associated with this initial data? Is it unique? Does it exist globally in time? Unfortunately, to this day there is no complete, satisfactory answer to this question in general. If the flow has one invariant direction (which is often physically unrealistic), then it has been known since the fundamental work of J. Leray ([57]) in 1934 that the equations are well-posed in the sense that for any finite energy initial data there is a unique, global-in-time solution, which has decreasing energy. On the other hand, in three space dimensions the situation is less clear. One can solve the equations (uniquely and globally in time) if the initial data is small enough, where the smallness is measured in a function space invariant under the scaling of the equations; see for instance [19, 35, 48, 50, 65. If the data is large, there are (possibly nonunique) global solutions of finite energy [56], which solve the equation in the sense of distributions, but uniqueness and smoothness are only known to hold for a short time. We shall not describe more in detail the numerous contributions on the question of the resolution of the (NS) equations but rather refer to [5, 54,55] for surveys on the Cauchy problem for (NS).

\subsection{Boltzmann: A mesoscopic point of view.}

2.3.1. The equations. L. Boltzmann's equation goes back to 1872 . It can be understood as an intermediate step in the analysis of fluid motion, between Newton's microscopic approach and the Navier-Stokes macroscopic description. The idea is to place the description at a more statistical level, describing the number, or density, of microscopic particles, which at a time $t$ have position $x$ and velocity $v$. Denoting this quantity by the probability density $f=f(t, x, v)$, Boltzmann's equation (introduced in [14,15]) states that $f$ evolves following

$$
\underbrace{\partial_{t} f+v \cdot \nabla_{x} f}_{\text {free transport }}=\underbrace{\alpha Q(f, f)}_{\text {localized binary collisions }}
$$


where the parameter $\alpha$ is the inverse of the mean free path of the microscopic particles and keeps track of the collision rate. The Boltzmann collision operator present in the right-hand side of (B) is the quadratic form, acting on the velocity variable, associated with the bilinear operator

$$
Q(f, f):=\iint_{\mathbb{S}^{d-1} \times \mathbb{R}^{d}}\left[f^{\prime} f_{1}^{\prime}-f f_{1}\right]\left(\left(v-v_{1}\right) \cdot \omega\right)_{+} d v_{1} d \omega
$$

where $\omega$ is the deflection angle, and we have used the standard abbreviations

$$
f=f(t, x, v), \quad f^{\prime}=f\left(t, x, v^{\prime}\right), \quad f_{1}^{\prime}=f\left(t, x, v_{1}^{\prime}\right), \quad f_{1}=f\left(t, x, v_{1}\right),
$$

with $\left(v^{\prime}, v_{1}^{\prime}\right)$ given, as in (2.3), by

$$
v^{\prime}=v+\omega \cdot\left(v_{1}-v\right) \omega, \quad v_{1}^{\prime}=v_{1}-\omega \cdot\left(v_{1}-v\right) \omega .
$$

The Boltzmann collision operator can be split into a gain term and a loss term (see 20,79])

$$
Q(f, f)=Q^{+}(f, f)-Q^{-}(f, f) .
$$

The loss term $Q^{-}$counts all collisions in which a given particle of (incoming) velocity $v$ will encounter another particle, of velocity $v_{1}$, and thus will change its velocity leading to a loss of particles of velocity $v$; whereas the gain term $Q^{+}$measures the number of particles of (outgoing) velocity $v$ which are created due to a collision between particles of velocities $v^{\prime}$ and $v_{1}^{\prime}$.

2.3.2. Solving the Boltzmann equation. We shall not detail all the mathematical literature concerning the resolution of (B) but will merely recall its main properties. It is important to notice that formally for any test function $\varphi$, there holds with the notation (2.6),

$$
\int Q(f, f) \varphi d v=\frac{1}{4} \int\left[f^{\prime} f_{1}^{\prime}-f f_{1}\right]\left(\varphi+\varphi_{1}-\varphi^{\prime}-\varphi_{1}^{\prime}\right)\left(\left(v-v_{1}\right) \cdot \omega\right)_{+} d v d v_{1} d \omega .
$$

In particular,

$$
\int Q(f, f) \varphi d v=0
$$

for all regular enough $f$, if and only if $\varphi(v)$ is a collision invariant; i.e., $\varphi(v)$ is a linear combination of $\left\{1, v_{1}, \ldots, v_{d},|v|^{2}\right\}$ (recall (2.4)). Thus, successively multiplying the Boltzmann equation (B) by the collision invariants and then integrating in velocity yields formally the local conservation laws of mass, momentum, and energy,

$$
\partial_{t} \int_{\mathbb{R}^{d}} f\left(\begin{array}{c}
1 \\
v \\
\frac{|v|^{2}}{2}
\end{array}\right) d v+\nabla_{x} \cdot \int_{\mathbb{R}^{d}} f\left(\begin{array}{c}
v \\
v \otimes v \\
\frac{|v|^{2}}{2} v
\end{array}\right) d v=0 .
$$

This provides a link to a macroscopic description of the gas.

The other very important feature of the Boltzmann equation comes also from the symmetries of the collision operator. Disregarding integrability issues, we choose $\varphi=\log f$ and find

$$
\begin{aligned}
D(f) & :=-\int_{\mathbb{R}^{d}} Q(f, f) \log f d v \\
& =\frac{1}{4} \int_{\mathbb{T}^{d} \times \mathbb{R}^{d} \times \mathbb{S}_{1}^{d-1}}\left(f^{\prime} f_{1}^{\prime}-f f_{1}\right) \log \frac{f^{\prime} f_{1}^{\prime}}{f f_{1}}\left(\left(v-v_{1}\right) \cdot \omega\right)_{+} d v d v_{1} d \omega \geq 0 .
\end{aligned}
$$


The so-defined entropy dissipation is therefore a nonnegative functional and the total entropy $\int_{\mathbb{R}^{d}} f \log f d v$ is formally decreasing. This leads to the Boltzmann H-theorem, also known as second principle of thermodynamics, stating that the entropy is (at least formally) a Lyapunov functional for the Boltzmann equation,

$$
\partial_{t} \int_{\mathbb{R}^{d}} f \log f d v+\nabla_{x} \cdot \int_{\mathbb{R}^{d}} f \log f v d v \leq 0 .
$$

As to the equation $Q(f, f)=0$, it is possible to show that it is only satisfied by the so-called Maxwellian distributions $M_{\rho, u, \theta}$, which are defined by

$$
M_{\rho, u, \theta}(v):=\frac{\rho}{(2 \pi \theta)^{\frac{d}{2}}} e^{-\frac{|v-u|^{2}}{2 \theta}},
$$

where $\rho \in \mathbb{R}_{+}, u \in \mathbb{R}^{d}$, and $\theta \in \mathbb{R}_{+}$are, respectively, the macroscopic density, bulk velocity, and temperature, under some appropriate choice of units. Maxwellian distributions are therefore stationary states; their entropy in particular does not decrease. In the following we set

$$
M_{\beta}(v):=\left(\frac{\beta}{2 \pi}\right)^{\frac{d}{2}} e^{-\frac{\beta|v|^{2}}{2}} \text { and } M(v):=M_{1}(v) .
$$

Concerning the Cauchy problem, the theory is far from being complete, similarly to the three-dimensional Navier-Stokes equations recalled in the previous section: global existence of weak (actually renormalized) solutions is known to hold [29], but uniqueness is only known in the case when the initial data is small enough (and decaying sufficiently fast at infinity in velocity space); see for instance [76, 77].

2.4. Hilbert's sixth problem. At the second International Congress of Mathematicians held in Paris in 1900, D. Hilbert presented his famous list of twenty-three open questions [4]. Some of those questions have since been solved, and some remain open to this day. Among these, we are interested here in the following question: to develop "mathematically the limiting processes [...] which lead from the atomistic view to the laws of motion of continua".

Our aim in this survey is to present some some mathematical progress that has been made recently on this question. Note that an answer to this question contains in particular an explanation to the appearance of irreversibility when passing from one description to the other, since the system of hard-spheres (2.1)-(2.3) is timereversible while the Boltzmann equation (B) and the Navier-Stokes equations (NS) are not.

\section{Some mathematical ATtempts AT RECONCILING SCALES}

3.1. Introduction. In the large $N$ limit, individual trajectories become irrelevant, and our goal is to describe an average behavior. This average is of course over particles which are indistinguishable. Because we have only a vague knowledge of the state of the system at initial time, we average over initial configurations. At time 0 , we thus start with a distribution $f_{N}^{0}\left(Z_{N}\right)$, where we use the notation introduced in Section 2.1 and we define a probability $f_{N}=f_{N}\left(t, Z_{N}\right)$, referred to as the $N$-particle distribution function. We assume that it satisfies for all permutations $\sigma$ of $\{1, \ldots, N\}$,

$$
f_{N}\left(t, Z_{\sigma(N)}\right)=f_{N}\left(t, Z_{N}\right)
$$


with $Z_{\sigma(N)}=\left(x_{\sigma(1)}, v_{\sigma(1)}, \ldots, x_{\sigma(N)}, v_{\sigma(N)}\right)$. Since $f_{N}$ is an invariant of the particle system, the Liouville equation relative to the particle system (2.1) is

$$
\partial_{t} f_{N}+\sum_{i=1}^{N} v_{i} \cdot \nabla_{x_{i}} f_{N}=0
$$

on the domain $\mathcal{D}_{N}^{\varepsilon}$ defined in (2.2), with the boundary condition

$$
f_{N}\left(t, Z_{N}^{\text {in }}\right)=f_{N}\left(t, Z_{N}^{\text {out }}\right)
$$

meaning that on the part of the boundary of $\mathcal{D}_{N}^{\varepsilon}$ such that $\left|x_{i}-x_{j}\right|=\varepsilon$, there holds

$$
f_{N}\left(t, \ldots, x_{i}, v_{i}^{\text {out }}, \ldots, x_{j}, v_{j}^{\text {out }}, \ldots\right)=f_{N}\left(t, \ldots, x_{i}, v_{i}^{\text {in }}, \ldots, x_{j}, v_{j}^{\text {in }}, \ldots\right),
$$

where the ingoing and outgoing velocities are related by (2.3).

3.2. A direct approach from microscopic to macroscopic scales. A natural approach to derive fluid mechanics equations from particle systems is to start with the following empirical distributions acting on $\mathbb{R}^{+} \times \mathbb{T}^{d}$ :

$$
\begin{aligned}
& \rho_{N}(t, x):=\frac{1}{N} \sum_{i=1}^{N} \delta_{x-x_{i}(t)}, \\
& u_{N}(t, x):=\frac{1}{N} \sum_{i=1}^{N} v_{i}(t) \delta_{x-x_{i}(t)}, \\
& e_{N}(t, x):=\frac{1}{2 N} \sum_{i=1}^{N}\left|v_{i}(t)\right|^{2} \delta_{x-x_{i}(t)},
\end{aligned}
$$

and to try to use laws of large numbers or large deviation principles to obtain their limiting behavior as $N$ goes to infinity. For a relation between the $N$-particle distribution function $f_{N}$ (which is a joint distribution of the system of $N$ indistinguishable particles) and empirical distributions (probability measures on the 1-particle phase space) and their projections in physical space defined above, we refer for instance to 38 . This has been achieved successfully in some asymptotic regimes, in the case when noise is added to the microscopic system. We refer for instance to 64 for a derivation of the Euler equations when the momenta of nearby particles are exchanged stochastically (with a noise of very small amplitude) or to [33, 67. for a derivation of the incompressible Navier-Stokes equations. We shall not give more detail here as our goal is to derive fluid mechanics equations from deterministic particle systems, and to this day the direct approach starting from empirical distributions, with no additional randomness, seems out of reach.

3.3. From mesoscopic to macroscopic scales. Starting from the Boltzmann equation (B), it is possible to derive formally a number of (though not all classes of) fluid mechanics equations. The formal method goes back to Hilbert 44, and Chapman and Enskog [23, and it consists in looking for asymptotic expansions in terms of $\alpha$. More precisely, expanding the solution $f$ to (B) under the form

$$
f(t, z)=\sum_{n \geq 0} \alpha^{-n} f_{n}(t, z)
$$

recalling that $z=(x, v)$, plugging the expansion into (B), and identifying powers of $\alpha$ formally gives rise to the Euler equations as well as the weakly viscous 
incompressible Navier-Stokes equations at the next order (but also at higher orders for other equations such as the Burnett model). Using truncated asymptotic expansions, it has been possible [18,51] to obtain a a rigorous justification of the compressible Euler limit up to the first singular time for the solution of the Euler system, and similarly for the Navier-Stokes system [24]. In [6, 7, Bardos, Golse, and Levermore devised a program for deriving weak solutions of the Navier-Stokes equations (NS) from the DiPerna-Lions solutions of the Boltzmann equation (B). One of the fundamental ideas behind this program is that the proof should only require a priori estimates coming from physics (namely mass, energy, and entropy bounds). The difficulty in the approach however lies in the very poor understanding of renormalized solutions. Nevertheless, F. Golse and L. Saint-Raymond [39,40] were able to achieve this program in the diffusive scaling limit. The precise statement, in three space dimensions, is the following.

Theorem 3.1 (39,40]). Consider a family $\left(f_{\alpha, 0}\right)_{\alpha>1}$ of initial data, the relative entropy of which satisfies, uniformly in $\alpha$,

$$
\frac{1}{\alpha^{2}} \int f_{\alpha, 0} \log \left(\frac{f_{\alpha, 0}}{M}\right) d x d v \leq C_{0}
$$

and such that as $\alpha$ goes to infinity the following limits hold in the sense of distributions

$$
\frac{1}{\alpha} P \int f_{\alpha, 0} v d v \rightarrow u_{0} \quad \text { and } \quad \frac{1}{\alpha} \int\left(f_{\alpha, 0}-M\right)\left(\frac{1}{5}|v|^{2}-1\right) d v \rightarrow \theta_{0},
$$

where $P$ denotes the projection onto divergence-free vector fields.

If $f_{\alpha}=M\left(1+\frac{1}{\alpha} g_{\alpha}\right)$ is an associate family of renormalized solutions to the scaled Boltzmann equation

$$
\frac{1}{\alpha} \partial_{t} f+v \cdot \nabla_{x} f=\alpha Q(f, f)
$$

then $g_{\alpha}$ is relatively weakly compact in $L_{\text {loc }}^{1}\left(d t d x ; L^{1}\left(\left(1+|v|^{2}\right) M d v\right)\right)$ as $\alpha$ goes to infinity, and any limit point $g$ of $g_{\alpha}$ can be written as

$$
g=\rho+u \cdot v+\theta \frac{|v|^{2}-3}{2},
$$

where $u$ satisfies the incompressible Navier-Stokes equations with data $u_{0}$ and $\rho, \theta$ are linked by the Fourier system

$$
\partial_{t} \theta+u \cdot \nabla \theta-\kappa \Delta \theta=0, \quad \theta_{\mid t=0}=\theta_{0}, \quad \text { and } \nabla(\rho+\theta)=0 .
$$

The viscosities can be explicitly computed.

The proof of this result is difficult and will not be described in these notes. Let us simply mention that it relies on an approach known as the moment method going back to [7] and [58, and one of the main difficulties in implementing this method is to control large velocities while gaining some equi-integrability properties in the space variable. That is the main achievement of [39, 40, following an idea of 68]; see also [69].

In Section 4 we describe the proof of a considerably simpler result, going from a linear scaled Boltzmann equation to the heat equation: this is by no means intended as an explanation of the proof of Theorem 3.1 but it will give an idea of the reason why a diffusive equation appears as an asymptotic regime for the Boltzmann equation. 
3.4. From microscopic to mesoscopic scales. The previous paragraph showed that it is possible to derive the incompressible Navier-Stokes equations from the nonlinear Boltzmann equation as $\alpha$ goes to infinity, in diffusive times (see Theorem 3.1). The question is now about deriving the Boltzmann equation from particle systems, as putting both arguments together should provide a complete derivation of the incompressible Navier-Stokes equations from particle systems. As we shall see in this paragraph, this part of the program remains largely unsolved.

We shall present the strategy of Lanford [52, which is essentially the only one known to this day (we refer the interested reader to a variant introduced in 60] via a semigroup approach to the study of the probability of trees). It consists in studying the asymptotics of the first marginal $f_{N}^{(1)}$ of the distribution function $f_{N}$, defined by

$$
f_{N}^{(1)}\left(t, z_{1}\right):=\int f_{N}\left(t, Z_{N}\right) d z_{2} \cdots d z_{N}
$$

More generally, we define the marginal of order $s \in[1, N]$ by

$$
f_{N}^{(s)}\left(t, Z_{s}\right):=\int f_{N}\left(t, Z_{N}\right) d z_{s+1} \cdots d z_{N}
$$

It corresponds to the joint probability of $s$-particles. Notice that for example in the particular case of tensorized (or chaotic, see the statement of Theorem 3.2 below) functions

$$
f_{N}\left(t, Z_{N}\right):=\prod_{i=1}^{N} f\left(t, z_{i}\right)
$$

with $f$ a probability, there holds simply

$$
f_{N}^{(s)}\left(t, Z_{s}\right)=\prod_{i=1}^{s} f\left(t, z_{i}\right) .
$$

Lanford's theorem is the following.

Theorem $3.2([52])$. Consider a system of $N$ hard-spheres of diameter $\varepsilon$ on the $d$ dimensional periodic box $\mathbb{T}^{d}=[0,1]^{d}$ (with $d \geq 2$ ), initially independent in the sense that

$$
f_{N, 0}\left(Z_{N}\right)=\frac{1}{\mathcal{Z}_{N}} \prod_{i=1}^{N} f_{0}\left(z_{i}\right) \prod_{k \neq j} \mathbf{1}_{\left|x_{k}-x_{j}\right|>\varepsilon},
$$

where $f_{0}$ is a continuous density such that

$$
\left\|f_{0} \exp \left(\mu+\frac{\beta}{2}|v|^{2}\right)\right\|_{L^{\infty}\left(\mathbb{T}_{x}^{d} \times \mathbb{R}_{v}^{d}\right)} \leq 1
$$

for some $\beta>0, \mu \in \mathbb{R}$. We have denoted by $\mathcal{Z}_{N}$ the partition function, that is the normalizing constant for $f_{N, 0}$ to be a probability.

In the limit $N \rightarrow \infty$ with $N \varepsilon^{d-1}=\alpha$, the one particle distribution $f_{N}^{(1)}$ converges almost everywhere to the solution of the Boltzmann equation (B) with initial data $f_{0}$, on a time interval $\left[0, t^{*} / \alpha\right]$ where $t^{*}$ depends only on the parameters $\beta, \mu$. 
Remark 3.3.

- The statement and main steps of the proof of Theorem 3.2 go back to [52]. We refer also to [21], 22], and [36] for details of the proof.

- The limit $N \rightarrow \infty$ with $N \varepsilon^{d-1}=\alpha$ is known as the Boltzmann-Grad, or low density, limit (see [43]). It corresponds to a dilute gas since the volume occupied by the gas $N \varepsilon^{d}$ goes to zero as $N$ goes to infinity. On average a particle of given speed has $O(\alpha)$ collisions in a given time.

- The main drawback of the statement lies of course on the time interval on which the convergence is proved. Recall indeed that our aim is to take the limit $\alpha \rightarrow \infty$ to recover fluid mechanics equations, and that is impossible with Theorem 3.2 since the life span shrinks to zero in that limit. Note that in the case when the initial distribution is close to vacuum, it is possible to extend this result globally in time [46, 47].

Sketch of proof of Theorem 3.2, Let us explain the strategy of the proof, which is due to [52. The details are rather long and technical, and we refer the interested reader to [36] for instance.

It is not difficult to check that $f_{N}^{(1)}=f_{N}^{(1)}\left(t, x_{1}, v_{1}\right)$ satisfies the equation

$$
\partial_{t} f_{N}^{(1)}+v_{1} \cdot \nabla_{x_{1}} f_{N}^{(1)}=\mathcal{C}_{1,2} f_{N}^{(2)},
$$

where

$$
\mathcal{C}_{1,2}=\mathcal{C}_{1,2}^{+}-\mathcal{C}_{1,2}^{-}
$$

and

$$
\begin{array}{r}
\left(\mathcal{C}_{1,2}^{ \pm} f_{N}^{(2)}\right)\left(z_{1}\right):=(N-1) \varepsilon^{d-1} \int_{\mathbb{S}_{1}^{d-1} \times \mathbb{R}^{d}} f_{N}^{(2)}\left(x_{1}, v_{1}, x_{1}+\varepsilon \omega, v_{2}\right) \\
\times\left(\omega \cdot\left(v_{2}-v_{1}\right)\right)_{ \pm} d \omega d v_{2},
\end{array}
$$

the index "+" corresponding to postcollisional configurations and the index "-" to precollisional configurations. The boundary condition (3.3) imposes that

$$
\begin{array}{r}
\left(\mathcal{C}_{1,2}^{+} f_{N}^{(2)}\right)\left(z_{1}\right)=(N-1) \varepsilon^{d-1} \int_{\mathbb{S}_{1}^{d-1} \times \mathbb{R}^{d}} f_{N}^{(2)}\left(x_{1}, v_{1}^{\prime}, x_{1}+\varepsilon \omega, v_{2}^{\prime}\right) \\
\times\left(\omega \cdot\left(v_{2}-v_{1}\right)\right)_{+} d \omega d v_{2},
\end{array}
$$

where

$$
v_{1}^{\prime}:=v_{1}-\omega \cdot\left(v_{1}-v\right) \omega, \quad v_{2}^{\prime}:=v_{2}-\omega \cdot\left(v_{2}-v\right) \omega .
$$

On the other hand after a change of variables $\omega \mapsto-\omega$, one finds

$$
\begin{array}{r}
\left(\mathcal{C}_{1,2}^{-} f_{N}^{(2)}\left(Z_{2}\right):=(N-1) \varepsilon^{d-1} \int_{\mathbb{S}_{1}^{d-1} \times \mathbb{R}^{d}} f_{N}^{(2)}\left(x_{1}, v_{1}, x_{1}-\varepsilon \omega, v_{2}\right)\right. \\
\times\left(\omega \cdot\left(v_{2}-v_{1}\right)\right)_{+} d \omega d v_{2},
\end{array}
$$

so that

$$
\begin{aligned}
\left(\mathcal{C}_{1,2} f_{N}^{(2)}\right)\left(z_{1}\right)=(N-1) \varepsilon^{d-1} \int_{\mathbb{S}_{1}^{d-1} \times \mathbb{R}^{d}}\left(f_{N}^{(2)}\left(x_{1}, v_{1}^{\prime}, x_{1}+\varepsilon \omega, v_{s+1}^{\prime}\right)\right. \\
\left.-f_{N}^{(2)}\left(x_{1}, v_{1}, x_{1}-\varepsilon \omega, v_{1}\right)\right)\left(\omega \cdot\left(v_{2}-v_{1}\right)\right)_{+} d \omega d v_{2} .
\end{aligned}
$$

Notice that the process of transforming (3.7) into (3.9) may seem arbitrary, but it is actually not since the value of $f_{N}^{(2)}$ at outgoing configurations is prescribed by the boundary condition (3.3) so the transformation (3.8) is actually not optional. 
Assuming that $f_{N}^{(1)}$ has a limit $f$ when $N \rightarrow \infty$ under the Boltzmann-Grad scaling $N \varepsilon^{d-1}=\alpha$, and similarly for $f_{N}^{(2)}$ (which we denote $f^{(2)}$ ), we find that formally

where

$$
\partial_{t} f+v_{1} \cdot \nabla_{x_{1}} f=\mathcal{C}_{1,2}^{0} f^{(2)}
$$

with

$$
\mathcal{C}_{1,2}^{0}=\mathcal{C}_{1,2}^{0+}-\mathcal{C}_{1,2}^{0-}
$$

$$
\begin{array}{r}
\left(\mathcal{C}_{1,2}^{0, \pm} f^{(2)}\right)\left(z_{1}\right):=\int_{\mathbb{S}_{1}^{d-1} \times \mathbb{R}^{d}}\left(f^{(2)}\left(x_{1}, v_{1}^{\prime}, x_{1}, v_{2}^{\prime}\right)-f^{(2)}\left(x_{1}, v_{1}, x_{1}, v_{2}\right)\right) \\
\times\left(\omega \cdot\left(v_{2}-v_{1}\right)\right)_{+} d \omega d v_{2} .
\end{array}
$$

To conclude, one now assumes that

$$
f^{(2)}\left(x_{1}, v_{1}, x_{2}, v_{2}\right)=f\left(x_{1}, v_{1}\right) f\left(x_{2}, v_{2}\right),
$$

which is known as the propagation of chaos assumption, and the Boltzmann equation (B) appears immediately.

The difficulty in transforming the above argument into a rigorous proof lies in the justification of the different limits taken above, as well as on the justification of the propagation of chaos (3.10). Let us explain Lanford's main ideas (which were subsequently developed and made precise by, among others, [21, 22, 25, 36, 73, 74]). First, since the equation (3.6) on $f_{N}^{(1)}$ involves $f_{N}^{(2)}$, one needs to write the whole hierarchy of equations known as the BBGKY hierarchy (for N. Bogoliubov [13], M. Born and H. S. Green [16, J. G. Kirkwood [9], and J. Yvan 80])

$$
\partial_{t} f_{N}^{(s)}+\sum_{1 \leq i \leq s} v_{i} \cdot \nabla_{x_{i}} f_{N}^{(s)}=\mathcal{C}_{s, s+1} f_{N}^{(s+1)}
$$

where as above one can write

$$
\mathcal{C}_{s, s+1}=\sum_{i=1}^{s} \mathcal{C}_{s, s+1}^{i}
$$

where the index $i$ refers to the index of the interaction particle among the $s$ fixed particles, with the notation

$$
\begin{aligned}
\left(\mathcal{C}_{s, s+1}^{i} f_{N}^{(s+1)}\right)\left(Z_{s}\right):= & (N-s) \varepsilon^{d-1} \int_{\mathbb{S}_{1}^{d-1} \times \mathbb{R}^{d}} \int\left(\omega \cdot\left(v_{s+1}-v_{i}\right)\right)_{+} \\
& \times\left(f_{N}^{(s+1)}\left(z_{1}, \ldots, x_{i}, v_{i}^{\prime}, \ldots, z_{s}, x_{i}+\varepsilon \omega, v_{s+1}^{\prime}\right)\right. \\
& \left.\quad-f_{N}^{(s+1)}\left(Z_{s}, x_{i}-\varepsilon \omega, v_{s+1}\right)\right) d \omega d v_{s+1} .
\end{aligned}
$$

Denote by $\boldsymbol{\Psi}_{s}(t)$ the $s$-particle flow associated with the hard-spheres system, and by $\mathbf{S}_{s}$ the associated solution operator

$$
\mathbf{S}_{s}(t): \quad f \in L^{\infty}\left(\mathcal{D}_{s}^{\varepsilon} ; \mathbb{R}\right) \mapsto f\left(\mathbf{\Psi}_{s}(-t, \cdot)\right) \in L^{\infty}\left(\mathcal{D}_{s}^{\varepsilon} ; \mathbb{R}\right) .
$$

The time-integrated form of equation (3.11) is

$$
f_{N}^{(s)}\left(t, Z_{s}\right)=\mathbf{S}_{s}(t) f_{N}^{(s)}\left(0, Z_{s}\right)+\int_{0}^{t} \mathbf{S}_{s}(t-\tau) \mathcal{C}_{s, s+1} f_{N}^{(s+1)}\left(\tau, Z_{s}\right) d \tau .
$$

Notice that actually the only way to make sense of the collision operators is to use the above Duhamel formulation consisting in applying a transport operator to lift the singularity of the collision integral (where a trace on a hypersurface is taken); 
we refer to [36] for details. The total flow and total collision operators $\mathbf{S}$ and $\mathbf{C}_{N}$ are defined on finite sequences $G_{N}=\left(g_{s}\right)_{1 \leq s \leq N}$ as

$$
\left\{\begin{array}{l}
\forall s \leq N,\left(\mathbf{S}(t) G_{N}\right)_{s}:=\mathbf{S}_{s}(t) g_{s}, \\
\forall s \leq N-1,\left(\mathbf{C}_{N} G_{N}\right)_{s}:=\mathcal{C}_{s, s+1} g_{s+1}, \quad\left(\mathbf{C}_{N} G_{N}\right)_{N}:=0 .
\end{array}\right.
$$

We finally define solutions to the BBGKY hierarchy to be solutions of

$$
F_{N}(t)=\mathbf{S}(t) F_{N}(0)+\int_{0}^{t} \mathbf{S}(t-\tau) \mathbf{C}_{N} F_{N}(\tau) d \tau, \quad F_{N}=\left(f_{N}^{(s)}\right)_{1 \leq s \leq N} .
$$

The main idea is then to define a limit hierarchy by formally taking the limit $N \rightarrow \infty$ under the Boltzmann-Grad scaling $N \varepsilon^{d-1}=\alpha$. We thus define the limiting collision operators

$$
\mathcal{C}_{s, s+1}^{0}=\sum_{i=1}^{s} \mathcal{C}_{s, s+1}^{0, i}
$$

with

$$
\begin{aligned}
\mathcal{C}_{s, s+1}^{0, i} f^{(s+1)}\left(t, Z_{s}\right) & \\
:= & \alpha \int\left(\omega \cdot\left(v_{s+1}-v_{i}\right)\right)_{+} \\
& \quad \times\left(f^{(s+1)}\left(t, x_{1}, v_{1}, \ldots, x_{i}, v_{i}^{\prime}, \ldots, x_{s}, v_{s}, x_{i}, v_{s+1}^{\prime}\right)\right. \\
& \left.\quad-f^{(s+1)}\left(t, Z_{s}, x_{i}, v_{s+1}\right)\right) d \omega d v_{s+1} .
\end{aligned}
$$

As in (3.12), we can then define the total Boltzmann flow and collision operators $\mathbf{S}^{0}$ and $\mathbf{C}^{0}$ as

$$
\left\{\begin{array}{l}
\forall s \geq 1,\left(\mathbf{S}^{0}(t) G\right)_{s}:=\mathbf{S}_{s}^{0}(t) g_{s}, \\
\forall s \geq 1,\left(\mathbf{C}^{\mathbf{0}} G\right)_{s}:=\mathcal{C}_{s, s+1}^{0} g_{s+1},
\end{array}\right.
$$

so that solutions to the Boltzmann hierarchy solve

$$
F(t)=\mathbf{S}^{0}(t) F(0)+\int_{0}^{t} \mathbf{S}^{0}(t-\tau) \mathbf{C}^{0} F(\tau) d \tau, \quad F=\left(f^{(s)}\right)_{s \geq 1} .
$$

The crucial point is to notice that if

$$
f^{(s)}\left(t, Z_{s}\right)=\prod_{i=1}^{s} f\left(t, z_{i}\right)
$$

(meaning $f^{(s)}(t)$ is tensorized), then $f$ satisfies the Boltzmann equation (B). It follows that the chaos property (3.10) will automatically be satisfied if one proves the convergence of one hierarchy to the other, as well as uniqueness for the limit hierarchy.

It turns out that the restriction on the time interval on which Theorem 3.2 holds is precisely due to the proof of the well-posedness of the hierarchy. Indeed the proof relies on a Cauchy-Kowalevskaya type argument (in the spirit of $62,63,78$ ) which completely misses the structure of the collision operators which are dealt with as if the nonlinear term in the Boltzmann equation was $f^{2}$ instead of $Q(f, f)$. We refer to [36] for details.

Once well-posedness is proved, the main difficulty in the proof of the convergence of one hierarchy to the other lies in the possibility of recollisions in the BBGKY flow, meaning that two particles that have collided in the past (directly or indirectly 
via collisions in chain with other particles) may collide again in the future and be deflected one by the other. This type of situation is impossible in the Boltzmann hierarchy, where each $\mathcal{C}_{s, s+1}^{0, i}$ term in the Duhamel formulation corresponds to a collision of a particle labeled $i$ with a fresh particle labeled $s+1$, and in between each collision there is free flow-particles finding themselves at the same place at some moment simply continue their trajectory without being deflected. Recollisions must be eliminated, and that turns out to be possible thanks to geometric arguments which are valid as long as the following hold:

- There are not too many particles at play (of the order of $\log N$ at most). It is therefore necessary to truncate the Duhamel sum, which expresses the number of particles that have actually interacted at time $t$, directly or indirectly. This is possible thanks to the well-posedness of the hierarchy, which provides the necessary a priori bounds on the Duhamel series.

- The velocities of the interacting particles are under control (at most of size $O(\log N)$ ). Truncating velocities is possible thanks to the a priori bounds.

- Collision times are not too close, namely in the iterated Duhamel formula $\left|t_{i}-t_{i+1}\right| \geq \delta$ where $\delta$ scales like a power of $\varepsilon$. This is possible thanks to a Lebesgue dominated convergence argument.

We refer to [36] for more details.

\section{Both Limits RECONCILED: LINEAR MODELS OF FLUIDS}

Summarizing the two previous paragraphs, the limit from the mesoscopic to the macroscopic description of fluids corresponds to taking $\alpha \rightarrow \infty$ in the Boltzmann equation (B) (and rescaling time), and it is known in some situations, namely near equilibrium or for weak (renormalized) solutions. On the other hand the limit from the microscopic to the mesoscopic description corresponds to taking $N \rightarrow \infty$ with $N \varepsilon^{d-1}=\alpha$, and it is known for small times only, of the order of $\alpha^{-1}$. This prevents it from combining both limits to go from particles to fluids.

There are however some (linear) cases where much progress on the Hilbert program has been made; we refer for instance to the works [17, 27, 28, 37]. The full program we are after has been achieved recently in two linear contexts [10, 11, namely in deriving the linear heat and in Stokes-Fourier equations. We describe briefly the case studied in 10 in this paragraph: in the case of a tagged particle in a background at equilibrium, it is proved in [10 that its distribution satisfies a heat equation at the limit $N \rightarrow \infty$, using the linear Boltzmann equation as an intermediate step (with a parameter $\alpha$ going slowly to infinity with $N$ ). The precise result is the following.

Theorem 4.1 ([10]). Consider the initial distribution

$$
f_{N}^{0}\left(Z_{N}\right):=\frac{1}{\mathcal{Z}_{N}} \rho^{0}\left(x_{1}\right) M_{\beta}^{\otimes N}\left(V_{N}\right) \prod_{k \neq j} \mathbf{1}_{\left|x_{k}-x_{j}\right|>\varepsilon},
$$

with $\mathcal{Z}_{N}$ the normalizing constant. Assume that $\rho^{0}$ belongs to $C^{0}\left(\mathbb{T}^{d}\right)$. Then for all $T>0$ and all $\tau \in[0, T]$, the distribution $f_{N}^{(1)}(\alpha \tau, x, v)$ remains close for the $L^{\infty}$ norm to $\rho(\tau, x) M_{\beta}(v)$ where $\rho(\tau, x)$ is the solution of the linear heat equation

$$
\partial_{\tau} \rho-\kappa_{\beta} \Delta_{x} \rho=0 \quad \text { in } \mathbb{T}^{d}, \quad \rho_{\mid \tau=0}=\rho^{0},
$$


and the diffusion coefficient $\kappa_{\beta}$ is given by

$$
\kappa_{\beta}:=\frac{1}{d} \int_{\mathbb{R}^{d}} v \mathcal{L}^{-1} v M_{\beta}(v) d v,
$$

where $\mathcal{L}$ is the linear Boltzmann operator (4.3) and $\mathcal{L}^{-1}$ is its pseudo-inverse defined on $(\operatorname{Ker} \mathcal{L})^{\perp}$. More precisely,

$$
\left\|f_{N}^{(1)}(\alpha \tau, x, v)-\rho(\tau, x) M_{\beta}(v)\right\|_{L^{\infty}\left([0, T] \times \mathbb{T}^{d} \times \mathbb{R}^{d}\right)} \rightarrow 0
$$

in the limit $N \rightarrow \infty$, with $\alpha=N \varepsilon^{d-1}$ going to infinity much slower than $\sqrt{\log \log N}$.

In the same asymptotic regime, the process $\Xi(\tau)=x_{1}(\alpha \tau)$ associated with the tagged particle converges in law toward a Brownian motion of variance $\kappa_{\beta}$, initially distributed under the measure $\rho^{0}$.

This is an extension of the works 9,53 , where the linear Boltzmann equation was derived for long times. We shall not describe further those results here, but simply mention that the main achievement consists in deriving the linear Boltzmann equation for an arbitrarily long time (contrary to the Lanford theorem which only holds for times of the order $\alpha^{-1}$ ), thanks to the use of the maximum principle associated with this very special type of initial data. To conclude this paragraph, we shall merely explain why the linear Boltzmann equation does have the heat equation as an asymptotic regime. Compared with Theorem 3.1, this can be considered as an exercise, but we feel it has some interest as it at least gives a flavor of the reason why a transport-type equation like (B) can lead asymptotically to a diffusive equation.

The linear Boltzmann equation is defined by linearizing the Boltzmann equation (B) around a Maxwellian $M_{\beta}$ and in dropping two of the four terms appearing in the linearization of the collision integral: factoring out the Maxwellian leads to the equation

$$
\begin{aligned}
& \partial_{t} \varphi_{\alpha}+v \cdot \nabla_{x} \varphi_{\alpha}=-\alpha \mathcal{L} \varphi_{\alpha}, \\
& \mathcal{L} \varphi_{\alpha}(v):=\iint\left[\varphi_{\alpha}(v)-\varphi_{\alpha}\left(v^{\prime}\right)\right] M_{\beta}\left(v_{1}\right)\left(\left(v-v_{1}\right) \cdot \omega\right)_{+} d v_{1} \omega .
\end{aligned}
$$

It is not difficult to prove that as soon as the initial data belongs to $L^{\infty}$, then there is a unique global solution to (4.3), which remains uniformly bounded in $\alpha$, for all times. The precise result describing the limit $\alpha \rightarrow \infty$ of $\varphi_{\alpha}$ in diffusive times is the following.

Proposition 4.2. Consider $\rho^{0}$ a continuous density of probability on $\mathbb{T}^{d}$, and let $\varphi_{\alpha}$ be the associate solution of (4.3) with initial data $\rho^{0}$. There holds for all $T \in[0, T]$

$$
\sup _{\tau \in[0, T]} \sup _{(x, v) \in \mathbb{T}^{d} \times \mathbb{R}^{d}}\left|M_{\beta}(v)\left(\varphi_{\alpha}(\alpha \tau, x, v)-\rho(\tau, x)\right)\right| \rightarrow 0, \quad \alpha \rightarrow \infty,
$$

where $\rho$ solves

$$
\partial_{\tau} \rho-\kappa_{\beta} \Delta_{x} \rho=0 \quad \text { in } \quad \mathbb{T}^{d}, \quad \rho_{\mid \tau=0}=\rho^{0},
$$

and the diffusion coefficient $\kappa_{\beta}$ is given by (4.1).

Main steps of the proof. Let us define

$$
\widetilde{\varphi}_{\alpha}(\tau, x, v):=\varphi_{\alpha}(\alpha \tau, x, v),
$$

which satisfies

$$
\partial_{\tau} \widetilde{\varphi}_{\alpha}+\alpha v \cdot \nabla_{x} \widetilde{\varphi}_{\alpha}+\alpha^{2} \mathcal{L} \widetilde{\varphi}_{\alpha}=0 .
$$


Notice that by the maximum principle on the heat equation, we may assume without loss of generality that $\rho^{0}$ is smooth. As recalled above, the formal Hilbert expansion consists in writing an asymptotic expansion of $\widetilde{\varphi}_{\alpha}$ in terms of powers of $\alpha^{-1}$,

$$
\widetilde{\varphi}_{\alpha}(\tau, x, v)=\widetilde{\rho}_{0}(\tau, x, v)+\frac{1}{\alpha} \widetilde{\rho}_{1}(\tau, x, v)+\frac{1}{\alpha^{2}} \widetilde{\rho}_{2}(\tau, x, v)+\cdots,
$$

in plugging that expansion into equation (4.5), and in canceling successively all the powers of $\alpha$. This gives formally the following set of equations, keeping only the $O(1), O(\alpha)$, and $O\left(\alpha^{2}\right)$ terms:

$$
\begin{aligned}
\mathcal{L} \widetilde{\rho}_{0} & =0, \\
v \cdot \nabla_{x} \widetilde{\rho}_{0}+\mathcal{L} \widetilde{\rho}_{1} & =0, \\
\partial_{\tau} \widetilde{\rho}_{0}+v \cdot \nabla_{x} \widetilde{\rho}_{1}+\mathcal{L} \widetilde{\rho}_{2} & =0 .
\end{aligned}
$$

In order to find the expressions for $\widetilde{\rho}_{1}$ and $\widetilde{\rho}_{2}$, as well as the equation on $\widetilde{\rho}_{0}$ (which we expect to be the heat equation), it is necessary to be able to invert the operator $\mathcal{L}$. It is known (see [44]) that $\mathcal{L}$ is invertible on the set of functions

$$
\left\{g \in L^{2}\left(\mathbb{R}^{d}, a_{\beta} M_{\beta} d v\right), \int_{\mathbb{R}^{d}} g(v) M_{\beta}(v) d v=0\right\},
$$

where

$$
a_{\beta}(v):=\int_{\mathbb{S}^{d-1} \times \mathbb{R}^{d}} M_{\beta}\left(v_{1}\right)\left(\left(v-v_{1}\right) \cdot \omega\right)_{+} d \omega d v_{1} .
$$

The first equation in (4.6) therefore reflects the fact that $\widetilde{\rho}_{0}$ does not depend on $v$. We next define the vector $b(v)=\left(b_{k}(v)\right)_{k \leq d}$ with $\int_{\mathbb{R}^{d}} b(v) M_{\beta}(v) d v=0$ by

$$
\mathcal{L} b(v):=v .
$$

Returning to (4.6), we have

$$
\widetilde{\rho}_{1}(\tau, x, v)=\rho_{1}(\tau, x, v)+\bar{\rho}_{1}(\tau, x),
$$

with

$$
\rho_{1}(\tau, x, v):=-b(v) \cdot \nabla_{x} \widetilde{\rho}_{0}(\tau, x) \quad \text { and } \quad \bar{\rho}_{1} \in \operatorname{Ker} \mathcal{L} .
$$

Next we consider the last equation in (4.6), and we notice that for $\widetilde{\rho}_{2}$ to exist it is necessary for $\partial_{\tau} \widetilde{\rho}_{0}+v \cdot \nabla_{x} \widetilde{\rho}_{1}$ to belong to the range of $\mathcal{L}$. Since $\widetilde{\rho}_{0}$ does not depend on $v$, this means that

$$
\partial_{\tau} \widetilde{\rho}_{0}+\int_{\mathbb{R}^{d}} v \cdot \nabla_{x} \widetilde{\rho}_{1}(\tau, x, v) M_{\beta}(v) d v=0 .
$$

We then define the diffusion matrix $D(v)=\left(D_{k, \ell}(v)\right)_{k, \ell \leq d}$ by

$$
\mathcal{L} D(v):=v \otimes b(v)-\int_{\mathbb{R}^{d}} v \otimes b(v) M_{\beta}(v) d v .
$$

From the symmetry of the model, one can check (see [26] for instance) that there is a function $\gamma$ such that

$$
b(v)=\gamma(|v|) v .
$$

The end of the formal proof is an easy computation, noticing that by symmetry of $b$

$$
\frac{1}{d} \int_{\mathbb{R}^{d}} v \mathcal{L}^{-1} v M_{\beta}(v) d v=\frac{1}{d} \int_{\mathbb{R}^{d}} \gamma(|v|)|v|^{2} M_{\beta}(v) d v
$$

Turning these formal arguments into a proof of convergence is not difficult and follows from the maximum principle. We refer for instance to [8,10, for details. 


\section{CONCLUSiOn AND OPEN PROBLEMS}

In this survey we have presented some recent mathematical results concerning the derivation of fluid mechanics equations from the fundamental laws of mechanics and have shown that the full derivation of the Navier-Stokes equations from Newton's laws is still widely open, due in particular to our lack of understanding of the derivation of the Boltzmann equation for large times. One of the reasons that this derivation fails to hold for large times (or large $\alpha$ ) is that to this day we are unable to use the fundamental property of the Boltzmann equation-namely the entropy dissipation - at the level of particles.

Setting this problem aside, there are still many other open problems, even in the short-time derivation of the Boltzmann equation.

- We have assumed that the particles are all identical spheres of mass 1 and diameter $\varepsilon>0$. This assumption could be slightly relaxed to masses of comparable size; however, if the masses, shapes, and sizes of the particles differ substantially, then some different phenomena may appear (see [12, $30,32,45$, for instance).

- We have assumed that the particles evolve in a periodic box of size 1 denoted $\mathbb{T}^{d}=[0,1]^{d}$. Other situations could be considered, like the whole space $\mathbb{R}^{d}$ - the absence of boundaries substantially simplifies the analysis. In particular, although the passage from Boltzmann to Navier-Stokes does hold in the presence of boundaries [59], essentially nothing is known in the derivation of the Boltzmann equation in a domain with boundaries - except of course if the problem can be reduced to the whole space or to a periodic box by symmetry.

- We have assumed that the particles interact elastically at each binary collision and there is no other type of interaction nor forcing. The case when more than two particles collide at the same time can be neglected rather easily (see Proposition 2.1). The case of more general interaction potentials is of course very interesting and physically relevant, but to this day this has only been considered under very stringent conditions on the potential [4, 36, 66.

Finally, we have focused on the derivation of the incompressible Navier-Stokes equations, but almost all remains to be done concerning the first limit of Boltzmann when $\alpha \rightarrow \infty$ (without rescaling in time), namely the compressible Euler equation; we refer to Section 3.3 for some comments.

\section{ABOUt THE AUTHOR}

Isabelle Gallagher is professor of mathematics at École Normale Supérieure (ENS) in Paris, on leave from Université Paris-Diderot. She is currently head of the department of mathematics at ENS. Her main research is in partial differential equations.

\section{REFERENCES}

[1] R. K. Alexander, The infinite hard-sphere system, ProQuest LLC, Ann Arbor, MI, 1975. Thesis (Ph.D.)-University of California, Berkeley. MR2625918

[2] R. Alexander, Time evolution for infinitely many hard spheres, Comm. Math. Phys. 49 (1976), no. 3, 217-232. MR0434284

[3] J. d'Alembert, Essai d'une nouvelle théorie de la résistance des fluides (1752), Paris. 
[4] N. Ayi, From Newton's law to the linear Boltzmann equation without cut-off, Comm. Math. Phys. 350 (2017), no. 3, 1219-1274, DOI 10.1007/s00220-016-2821-6. MR3607474

[5] H. Bahouri, J.-Y. Chemin, and R. Danchin, Fourier analysis and nonlinear partial differential equations, Grundlehren der Mathematischen Wissenschaften [Fundamental Principles of Mathematical Sciences], vol. 343, Springer, Heidelberg, 2011. MR2768550

[6] C. Bardos, F. Golse, and C.D. Levermore, Fluid dynamic limits of the Boltzmann equation I, J. Stat, Phys. 63 (1991), 323-344.

[7] C. Bardos, F. Golse, and C. D. Levermore, Fluid dynamic limits of kinetic equations. II. Convergence proofs for the Boltzmann equation, Comm. Pure Appl. Math. 46 (1993), no. 5, 667-753, DOI 10.1002/cpa.3160460503. MR.1213991

[8] C. Bardos, R. Santos, and R. Sentis, Diffusion approximation and computation of the critical size, Trans. Amer. Math. Soc. 284 (1984), no. 2, 617-649, DOI 10.2307/1999099. MR743736

[9] H. van Beijeren, O. E. Lanford III, J. L. Lebowitz, and H. Spohn, Equilibrium time correlation functions in the low-density limit, J. Statist. Phys. 22 (1980), no. 2, 237-257, DOI 10.1007/BF01008050. MR560556

[10] T. Bodineau, I. Gallagher, and L. Saint-Raymond, The Brownian motion as the limit of a deterministic system of hard-spheres, Invent. Math. 203 (2016), no. 2, 493-553, DOI 10.1007/s00222-015-0593-9. MR 3455156

[11] T. Bodineau, I. Gallagher, and L. Saint-Raymond, From hard sphere dynamics to the StokesFourier equations: an $L^{2}$ analysis of the Boltzmann-Grad limit, Ann. PDE 3 (2017), no. 1, Art. 2, 118, DOI 10.1007/s40818-016-0018-0. MR3625187

[12] T. Bodineau, I. Gallagher, and L. Saint-Raymond, Derivation of an Ornstein-Uhlenbeck process for a massive particle in a rarified gas of particles, Ann. Henri Poincaré 19 (2018), no. 6, 1647-1709, DOI 10.1007/s00023-018-0674-6. MR.3806440

[13] N. N. Bogoliubov, Problems of a dynamical theory in statistical physics, Studies in Statistical Mechanics, Vol. I, North-Holland, Amsterdam; Interscience, New York, 1962, pp. 1-118. MR.0136381

[14] L. Boltzmann, Weitere Studien uber das Warmegleichgenicht unfer Gasmolakular, Sitzungsberichte der Akademie der Wissenschaften 66 (1872), 275-370. Translation : Further studies on the thermal equilibrium of gas molecules, in Kinetic Theory 2, 88-174, Ed. S.G. Brush, Pergamon, Oxford (1966).

[15] L. Boltzmann, Leçons sur la théorie des gaz, Gauthier-Villars (Paris, 1902-1905). Ré-édition Jacques Gabay, 1987.

[16] M. Born and H. S. Green, A general kinetic theory of liquids. I. The molecular distribution functions, Proc. Roy. Soc. London. Ser. A. 188 (1946), 10-18, DOI 10.1098/rspa.1946.0093. MR.0023769

[17] L. A. Bunimovich and Ya. G. Sină, Statistical properties of Lorentz gas with periodic configuration of scatterers, Comm. Math. Phys. 78 (1980/81), no. 4, 479-497. MR606459

[18] R. E. Caflisch, The Boltzmann equation with a soft potential. I. Linear, spatiallyhomogeneous, Comm. Math. Phys. 74 (1980), no. 1, 71-95. MR.575897

[19] M. Cannone, A generalization of a theorem by Kato on Navier-Stokes equations, Rev. Mat. Iberoamericana 13 (1997), no. 3, 515-541, DOI 10.4171/RMI/229. MR.1617394

[20] C. Cercignani, The Boltzmann equation and its applications, Applied Mathematical Sciences, vol. 67, Springer-Verlag, New York, 1988. MR.1313028

[21] C. Cercignani, V. I. Gerasimenko, and D. Ya. Petrina, Many-particle dynamics and kinetic equations, Mathematics and its Applications, vol. 420, Kluwer Academic Publishers Group, Dordrecht, 1997. Translated from the Russian manuscript by K. Petrina and V. Gredzhuk. MR 1472233

[22] C. Cercignani, R. Illner, and M. Pulvirenti, The mathematical theory of dilute gases, Applied Mathematical Sciences, vol. 106, Springer-Verlag, New York, 1994. MR1307620

[23] S. Chapman and T. G. Cowling, The mathematical theory of non-uniform gases: An account of the kinetic theory of viscosity, thermal conduction, and diffusion in gases, Cambridge University Press, New York, 1960. MR0116537

[24] A. De Masi, R. Esposito, and J. L. Lebowitz, Incompressible Navier-Stokes and Euler limits of the Boltzmann equation, Comm. Pure Appl. Math. 42 (1989), no. 8, 1189-1214, DOI 10.1002/cpa.3160420810. MR.1029125 
[25] R. Denlinger, The propagation of chaos for a rarefied gas of hard spheres in the whole space, Arch. Ration. Mech. Anal. 229 (2018), no. 2, 885-952, DOI 10.1007/s00205-018-1229-1. MR.3803778

[26] L. Desvillettes and F. Golse, A remark concerning the Chapman-Enskog asymptotics, Advances in kinetic theory and computing, Ser. Adv. Math. Appl. Sci., vol. 22, World Sci. Publ., River Edge, NJ, 1994, pp. 191-203. MR1323184

[27] L. Desvillettes and M. Pulvirenti, The linear Boltzmann equation for long-range forces: a derivation from particle systems, Math. Models Methods Appl. Sci. 9 (1999), no. 8, 11231145, DOI 10.1142/S0218202599000506. MR 1722064

[28] L. Desvillettes and V. Ricci, A rigorous derivation of a linear kinetic equation of FokkerPlanck type in the limit of grazing collisions, J. Statist. Phys. 104 (2001), no. 5-6, 1173-1189, DOI 10.1023/A:1010461929872. MR 1859001

[29] R. J. DiPerna and P.-L. Lions, On the Cauchy problem for Boltzmann equations: global existence and weak stability, Ann. of Math. (2) 130 (1989), no. 2, 321-366, DOI 10.2307/1971423. MR.1014927

[30] M. Dobson, F. Legoll, T. Lelièvre, and G. Stoltz, Derivation of Langevin dynamics in a nonzero background flow field, ESAIM Math. Model. Numer. Anal. 47 (2013), no. 6, 15831626, DOI 10.1051/m2an/2013077. MR3110489

[31] D. Dürr, S. Goldstein, and J. L. Lebowitz, A mechanical model of Brownian motion, Comm. Math. Phys. 78 (1980/81), no. 4, 507-530. MR606461

[32] D. Dürr, S. Goldstein, and J. L. Lebowitz, A mechanical model for the Brownian motion of a convex body, Z. Wahrsch. Verw. Gebiete 62 (1983), no. 4, 427-448, DOI 10.1007/BF00534196. MR690569

[33] R. Esposito, R. Marra, and H. T. Yau, Navier-Stokes equations for stochastic particle systems on the lattice, Comm. Math. Phys. 182 (1996), no. 2, 395-456. MR 1447299

[34] L. Euler, Principles of the motion of fluids, Phys. D 237 (2008), no. 14-17, 1840-1854, DOI 10.1016/j.physd.2008.04.019. English adaptation by Walter Pauls. MR2449769

[35] H. Fujita and T. Kato, On the Navier-Stokes initial value problem. I, Arch. Rational Mech. Anal. 16 (1964), 269-315, DOI 10.1007/BF00276188. MR0166499

[36] I. Gallagher, L. Saint-Raymond, and B. Texier, From Newton to Boltzmann: hard spheres and short-range potentials, Zurich Lectures in Advanced Mathematics, European Mathematical Society (EMS), Zürich, 2013. MR3157048

[37] G. Gallavotti, Statistical mechanics, Texts and Monographs in Physics, Springer-Verlag, Berlin, 1999. A short treatise. MR,1707309

[38] F. Golse, The mean-field limit for the dynamics of large particle systems, Journées "Équations aux Dérivées Partielles", Univ. Nantes, Nantes, 2003, pp. Exp. No. IX, 47. MR2050595

[39] F. Golse and L. Saint-Raymond, The Navier-Stokes limit of the Boltzmann equation for bounded collision kernels, Invent. Math. 155 (2004), no. 1, 81-161, DOI 10.1007/s00222-0030316-5. MR2025302

[40] F. Golse and L. Saint-Raymond, The incompressible Navier-Stokes limit of the Boltzmann equation for hard cutoff potentials (English, with English and French summaries), J. Math. Pures Appl. (9) 91 (2009), no. 5, 508-552, DOI 10.1016/j.matpur.2009.01.013. MR2517786

[41] A. N. Gorban, Hilbert's sixth problem: the endless road to rigour, Philos. Trans. Roy. Soc. A 376 (2018), no. 2118, 20170238, 10, DOI 10.1098/rsta.2017.0238. MR3797486

[42] A. N. Gorban and I. Karlin, Hilbert's 6th problem: exact and approximate hydrodynamic manifolds for kinetic equations, Bull. Amer. Math. Soc. (N.S.) 51 (2014), no. 2, 187-246, DOI 10.1090/S0273-0979-2013-01439-3. MR3166040

[43] H. Grad, On the kinetic theory of rarefied gases, Comm. Pure Appl. Math. 2 (1949), 331-407, DOI 10.1002/cpa.3160020403. MR0033674

[44] D. Hilbert, Begründung der kinetischen Gastheorie (German), Math. Ann. 72 (1912), no. 4, 562-577, DOI 10.1007/BF01456676. MR:1511713

[45] R. Holley, The motion of a heavy particle in an infinite one dimensional gas of hard spheres, Z. Wahrscheinlichkeitstheorie und Verw. Gebiete 17 (1971), 181-219, DOI 10.1007/BF00536757. MR0283907

[46] R. Illner and M. Pulvirenti, Global validity of the Boltzmann equation for a two-dimensional rare gas in vacuum, Comm. Math. Phys. 105 (1986), no. 2, 189-203. MR849204 
[47] R. Illner and M. Pulvirenti, Global validity of the Boltzmann equation for two- and threedimensional rare gas in vacuum. Erratum and improved result: "Global validity of the Boltzmann equation for a two-dimensional rare gas in vacuum" [Comm. Math. Phys. 105 (1986), no. 2, 189-203; MR0849204 (88d:82061)] and "Global validity of the Boltzmann equation for a three-dimensional rare gas in vacuum" [ibid. 113 (1987), no. 1, 79-85; MR0918406 (89b:82052)] by Pulvirenti, Comm. Math. Phys. 121 (1989), no. 1, 143-146. MR985619

[48] T. Kato, Strong $L^{p}$-solutions of the Navier-Stokes equation in $\mathbf{R}^{m}$, with applications to weak solutions, Math. Z. 187 (1984), no. 4, 471-480, DOI 10.1007/BF01174182. MR760047

[49] J. G. Kirkwood, The statistical mechanical theory of transport processes I. General theory, Journal of Chemical Physics 14 (1946), 180-202.

[50] H. Koch and D. Tataru, Well-posedness for the Navier-Stokes equations, Adv. Math. 157 (2001), no. 1, 22-35, DOI 10.1006/aima.2000.1937. MR1808843

[51] M. Lachowicz, On the initial layer and the existence theorem for the nonlinear Boltzmann equation, Math. Methods Appl. Sci. 9 (1987), no. 3, 342-366, DOI 10.1002/mma.1670090127. MR.908596

[52] O. E. Lanford III, Time evolution of large classical systems, Dynamical systems, theory and applications (Rencontres, Battelle Res. Inst., Seattle, Wash., 1974), Springer, Berlin, 1975, pp. 1-111. Lecture Notes in Phys., Vol. 38. MR.0479206

[53] J. L. Lebowitz and H. Spohn, Steady state self-diffusion at low density, J. Statist. Phys. 29 (1982), no. 1, 39-55, DOI 10.1007/BF01008247. MR676928

[54] P. G. Lemarié-Rieusset, Recent developments in the Navier-Stokes problem, Chapman \& Hall/CRC Research Notes in Mathematics, vol. 431, Chapman \& Hall/CRC, Boca Raton, FL, 2002. MR 1938147

[55] P. G. Lemarié-Rieusset, The Navier-Stokes problem in the 21st century, CRC Press, Boca Raton, FL, 2016. MR.3469428

[56] J. Leray, Sur le mouvement d'un liquide visqueux emplissant l'espace (French), Acta Math. 63 (1934), no. 1, 193-248, DOI 10.1007/BF02547354. MR.1555394

[57] J. Leray, Étude de diverses équations intégrales non linéaires et de quelques problèmes que pose l'hydrodynamique, Journal de Mathématiques Pures et Appliquées, 12 (1933), 1-82.

[58] P.-L. Lions and N. Masmoudi, From the Boltzmann equations to the equations of incompressible fluid mechanics. I, II, Arch. Ration. Mech. Anal. 158 (2001), no. 3, 173-193, 195-211, DOI 10.1007/s002050100143. MR1842343

[59] N. Masmoudi and L. Saint-Raymond, From the Boltzmann equation to the Stokes-Fourier system in a bounded domain, Comm. Pure Appl. Math. 56 (2003), no. 9, 1263-1293, DOI 10.1002/cpa.10095. MR.1980855

[60] K. Matthies and F. Theil, A semigroup approach to the justification of kinetic theory, SIAM J. Math. Anal. 44 (2012), no. 6, 4345-4379, DOI 10.1137/120865598. MR3028562

[61] C. Navier, Mémoire sur les lois du mouvement des fluides, Mémoire de l'Académie des Sciences de l'Institut de France, 6 (1822), 375-394.

[62] L. Nirenberg, An abstract form of the nonlinear Cauchy-Kowalewski theorem, J. Differential Geometry 6 (1972), 561-576. Collection of articles dedicated to S. S. Chern and D. C. Spencer on their sixtieth birthdays. MR0322321

[63] T. Nishida, A note on a theorem of Nirenberg, J. Differential Geom. 12 (1977), no. 4, 629-633 (1978). MR512931

[64] S. Olla, S. R. S. Varadhan, and H.-T. Yau, Hydrodynamical limit for a Hamiltonian system with weak noise, Comm. Math. Phys. 155 (1993), no. 3, 523-560. MR.1231642

[65] F. Planchon, Asymptotic behavior of global solutions to the Navier-Stokes equations in $\mathbf{R}^{3}$, Rev. Mat. Iberoamericana 14 (1998), no. 1, 71-93, DOI 10.4171/RMI/235. MR1639283

[66] M. Pulvirenti, C. Saffirio, and S. Simonella, On the validity of the Boltzmann equation for short range potentials, Rev. Math. Phys. 26 (2014), no. 2, 1450001, 64, DOI 10.1142/S0129055X14500019. MR3190204

[67] J. Quastel and H.-T. Yau, Lattice gases, large deviations, and the incompressible NavierStokes equations, Ann. of Math. (2) 148 (1998), no. 1, 51-108, DOI 10.2307/120992. MR.1652971

[68] L. Saint-Raymond, From the BGK model to the Navier-Stokes equations (English, with English and French summaries), Ann. Sci. École Norm. Sup. (4) 36 (2003), no. 2, 271-317, DOI 10.1016/S0012-9593(03)00010-7. MR1980313 
[69] L. Saint-Raymond, Hydrodynamic limits of the Boltzmann equation, Lecture Notes in Mathematics, vol. 1971, Springer-Verlag, Berlin, 2009. MR2683475

[70] M. Slemrod, From Boltzmann to Euler: Hilbert's 6th problem revisited, Comput. Math. Appl. 65 (2013), no. 10, 1497-1501, DOI 10.1016/j.camwa.2012.08.016. MR3061719

[71] M. Slemrod, The problem with Hilbert's 6th problem, Math. Model. Nat. Phenom. 10 (2015), no. 3, 6-15, DOI 10.1051/mmnp/201510302. MR3371918

[72] M. Slemrod, Hilbert's sixth problem and the failure of the Boltzmann to Euler limit, Philos. Trans. Roy. Soc. A 376 (2018), no. 2118, 20170222, 12, DOI 10.1098/rsta.2017.0222. MR.3797494

[73] H. Spohn, Boltzmann hierarchy and Boltzmann equation, Kinetic theories and the Boltzmann equation (Montecatini, 1981), Lecture Notes in Math., vol. 1048, Springer, Berlin, 1984, pp. 207-220, DOI 10.1007/BFb0071883. MR740726

[74] H. Spohn, Large scale dynamics of interacting particles, Texts and Monographs in Physics, Springer-Verlag, Berlin/Heidelberg, 1991, 174 pp.

[75] G. Stokes, On the theories of internal friction of fluids in motion and of the equilibrium and motion of elastic solids, Trans. Camb. Phil. Soc. 8 (1845), 287-319.

[76] S. Ukai, On the existence of global solutions of mixed problem for non-linear Boltzmann equation, Proc. Japan Acad. 50 (1974), 179-184. MR0363332

[77] S. Ukai, Les solutions globales de l'équation de Boltzmann dans l'espace tout entier et dans le demi-espace (French, with English summary), C. R. Acad. Sci. Paris Sér. A-B 282 (1976), no. 6, Ai, A317-A320. MR0445138

[78] S. Ukai, The Boltzmann-Grad limit and Cauchy-Kovalevskaya theorem: Recent topics in mathematics moving toward science and engineering, Japan J. Indust. Appl. Math. 18 (2001), no. 2, 383-392, DOI 10.1007/BF03168581. MR1842918

[79] C. Villani, A review of mathematical topics in collisional kinetic theory, Handbook of mathematical fluid dynamics, Vol. I, North-Holland, Amsterdam, 2002, pp. 71-305, DOI 10.1016/S1874-5792(02)80004-0. MR.1942465

[80] J. Yvon, La théorie statistique des fluides et l'équation d'état, Actual. Sci. et Indust. 203 (Paris, Hermann), 1935.

Université Paris-Diderot, Sorbonne Paris Cité, France

Current address: DMA, École Normale Suptérieure de Paris, UMR 8553, France

Email address: gallagher@math.ens.fr 